\title{
Developments in the Design of Centrifugal Pendulum Vibration Absorbers
}

\author{
David E. Newland \\ University of Cambridge, Engineering Design Centre, Trumpington Street, Cambridge, CB2 1PZ, UK.
}

\begin{abstract}
(Received 30 January 2020; accepted 21 April 2020)
For over 60 years, the torsional vibration of reciprocating aircraft engines has been controlled by centrifugal pendulum vibration absorbers. Loose weights attached to an engine's crankshaft act as tuned-mass absorbers by oscillating at a frequency in proportion to rotational speed. More recently, similar loose masses have been attached to the flywheels of automobile engines. The need to achieve increased power from fewer cylinders, while reducing weight and improving economy, has exacerbated torsional vibration of the drive train. The dynamics of a wheel carrying many centrifugal pendulums of bifilar design has been the subject of a growing literature, but much less has been written about roller-type pendulums and about overall system performance. This paper is a new analysis of bifilar and roller systems and their design requirements. The current state of knowledge about practical design limitations is explained and the need for further research discussed.
\end{abstract}

\section{INTRODUCTION}

The use of a tuned-mass absorber to reduce harmonic vibration of fixed frequency is well-known. In the 1930s, this principle was extended to reducing the torsional vibration of machinery where there is excitation whose frequency increases with speed. Loose masses moving in a curved track or constrained by rollers to move in a curved path allow the irregular firing torque of a reciprocating engine to be resisted, at least in theory. There is a good introduction in Den Hartog's classic textbook. ${ }^{3}$ The bifilar or Sarazin type of centrifugal pendulum is used widely, while the roller or Salomon type still finds new applications. Examples are shown in Figs. 1(a) and 1(b).

These devices are basically tuned-mass absorbers. They serve to reduce the amplitude of a troublesome resonance by generating negative reaction forces at the pendulums' resonant frequency. Because centrifugal force acts to hold the pendulum or roller in its equilibrium position, their natural frequency is speed dependent. Each pendulum has a natural frequency that is proportional to engine speed. For example, a fourth-order pendulum, $n=4$, has a natural frequency of 4 times engine rotational speed. This is important because the excitation harmonics of a reciprocating engine increase in frequency in proportion to engine speed. When the rotational speed is such that the $n=4$ excitation coincides with a torsional natural frequency of the drive train assembly, large amplitude torsional vibration may occur. Properly working centrifugal pendulum absorbers, tuned to $n=4$, reduce the $n=4$ harmonic of excitation at all engine speeds, and therefore they reduce resonant torsional response to this harmonic.

The usual theory of pendulum vibration absorbers is a constant speed theory. In practice, matters are not so simple. At low rotational speed, centrifugal forces are insufficient to hold the moving masses close to their central positions. Instead they rattle within the available clearance. The name "Rattler" has been registered as a trademark for one particular device of the Salomon type. As engine speed increases, the loose masses are pulled into their central positions. If there is significant excitation, there may still not be enough centrifugal force to generate sufficiently large harmonic reaction forces and some rattling continues. Then, above a critical speed, the centrifugal pendulums overcome the excitation, and pull into synchronism with the torque harmonic to which they are tuned. Above this engine speed, they start to work properly, reducing the ampli- tude of crankshaft torsional vibration.

This paper reviews the constructional features of commonly used centrifugal pendulums and brings together published results that bear on their design. It includes new analysis of the response of roller pendulums which have received much less attention in the literature, although they are widely used. Figure 7 is the result of this new analysis and compares the torque-absorbing capacities of bifilar, roller, and tautochronic pendulums for the first time. Modelling all aspects of a reciprocating engine's torsional dynamics is extremely complex and most studies have been confined to single pendulums or to several pendulums attached to the same wheel. Results for two- and three-degree-of-freedom torsional systems are shown in Figs. 8-10. An intriguing detail is the response loops that occur. These are discussed in section 7. All the results are for steady operation at a constant speed. A comprehensive analytical treatment of the large-amplitude dynamic response of an accelerating engine with centrifugal pendulums is not yet feasible.

Nomenclature is listed at the start of Appendix 1.

\section{CONSTRUCTIONAL DETAILS}

In aircraft applications, bifilar pendulums may be attached to the webs of every crank, Fig 2(a). For automobile engines, it is more usual to attach pendulums, of either bifilar or roller design, to one of the wheels of a dual-mass flywheel, Fig 2(b). A single, solid flywheel is replaced by two co-axial wheels joined by a torsional spring. The engine's crankshaft is attached to the first wheel and the clutch is attached to the second wheel. Usually, vibration absorbers are attached to the second wheel.

Salomon or roller pendulums are usually wheel mounted, and there may be 6 or more rollers on a single wheel. One commercial off-the-shelf design has 9 rollers, with 6 rollers tuned to one excitation order and 3 to a different order, Fig. 2(d).

Most commercial centrifugal pendulum absorbers move in a circular arc with respect to their attachment wheel or crank. As pendulum amplitude becomes large (of the order of 30 degrees) its natural frequency begins to decrease. For this reason, pendulum geometry is usually set so that the pendulum's smallamplitude (linear) natural frequency is higher than its intended value when the pendulum is operating at its design amplitude. 


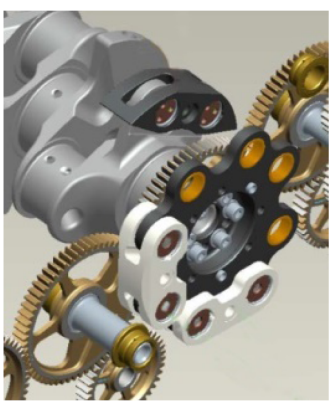

(a)

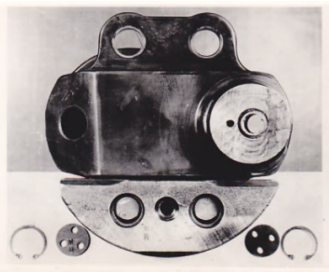

(c)

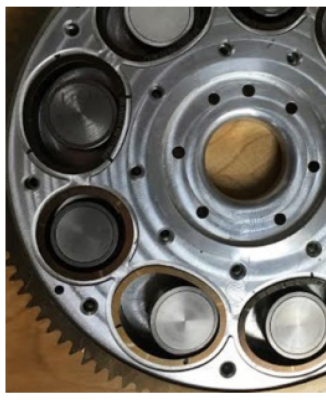

(b)

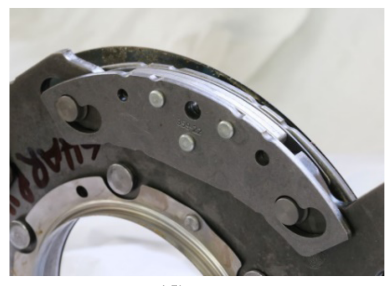

(d)
Figure 1. (a) Sarazin or bifilar pendulums (the white masses), (b) Salomon or roller pendulums, (c) Solid bifilar pendulum design, (d) laminated bifilar pendulum design.

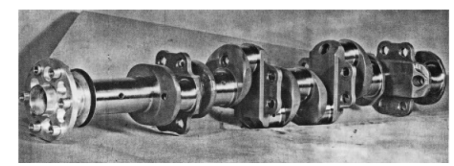

(a)

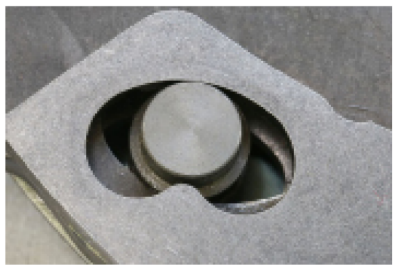

(c)

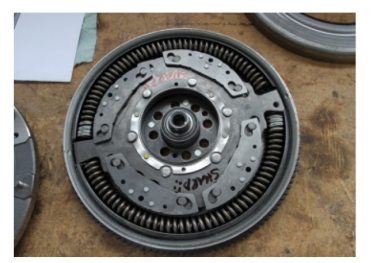

(b)

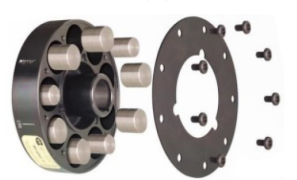

(d)
Figure 2. (a) 8-cylinder aircraft crankshaft with attachments for 8 bifilar pendulums, (b) gearbox side of automobile dualmass flywheel with 4 bifilar pendulums, (c) typical geometry of one side of a bifilar suspension, (d) TCI "Rattler ${ }^{\circledR}$ " rollertype absorber.

For a pendulum designed to absorb $n$th order vibration at rotational speed $\Omega$, the pendulum's natural frequency $\omega_{n}$ is usually set according to $\omega_{n}^{2}=(1+\epsilon) n^{2} \Omega^{2}$ where the detuning parameter $\epsilon$ is in the order of 0.1 . This is intended to ensure that $\omega_{n}$ is close to $n \Omega$ when the pendulum is working properly.

\section{DESIGN CALCULATIONS}

During design, the expected time-history of torque applied at each crank (of a multi-cylinder engine) must first be computed, including allowance for the reciprocating inertias. This data was then Fourier analysed to generate the amplitudes of each order of excitation torque and its variation over the required operating range of the engine. The major orders of excitation for a 4-cycle (4-stroke) engine depended on the number of cylinders. A one-cylinder engine has major order $n=1 / 2$ because there is one firing stroke every two revolutions. For an 8 -cylinder engine, it is $n=4$. Higher harmonics may also be important.
According to linear theory, if a pendulum is tuned precisely to an order of excitation, it will swing to enforce a nodal point for torsional vibration of the system to which it is attached. ${ }^{3}$ The centrifugal pendulum acts as an infinite inertia.

In practice, the response of a swinging pendulum is more complicated. If the pendulum's centre of mass moves in a circular arc, its natural frequency will decrease as amplitude increases. Although this can be compensated by changing the geometry from a circular arc, as pendulum amplitude increases, its reaction torque becomes increasingly nonlinear whatever its path. Eventually the fundamental component of pendulum reaction torque stops increasing, as other harmonics grow, and the pendulum no longer functions as a vibration absorber. There is therefore a practical limit on the reaction torque that a pendulum of given properties can generate.

\section{NONLINEAR CHARACTERISTICS}

When this limitation was first understood (see, for example, Newland 1964), nonlinear calculations allowed the maximum practical pendulum amplitude to be calculated. ${ }^{9}$ Usually a nonlinear jump occurs at an amplitude of about $60^{\circ}$ so that the design amplitude should be restricted to about $30^{\circ}$. Some authors have suggested only $10^{\circ}$ should be the design target (Mitchiner \& Leonard, 1991) but this seems to be unduly conservative. ${ }^{8}$

The reason for the amplitude limitation is illustrated in Fig. 3, based on the analysis in Appendix 2. Figure 3 shows (non-dimensional) pendulum torque calculated by equation (A2.1) for one complete cycle of a centrifugal pendulum swinging at different amplitudes. Harmonic oscillation of the pendulum with amplitudes of $30^{\circ}$ (black line), $60^{\circ}$ (blue) and $90^{\circ}$ (red), is illustrated in the upper left diagram. Corresponding pendulum torque is shown in the other three diagrams. For $30^{\circ}$ amplitude the pendulum torque was nearly harmonic. Its fundamental component is shown for comparison. For $60^{\circ}$ amplitude there was now significant deviation from the harmonic shape shown by the fundamental. For $90^{\circ}$ pendulum amplitude, pendulum torque was far from harmonic, as shown by its deviation from its fundamental component. The amplitude of the fundamental component of pendulum torque increased with increasing amplitude from $30^{\circ}$ to $60^{\circ}$ but decreased as the amplitude rose towards $90^{\circ}$. Then the third harmonic dominated and the pendulum's vibration absorbing property had transferred from order $n$ to order $3 n$, with the pendulum no longer absorbing the intended component of torsional vibration.

By altering the path that the pendulum mass swung through from a circular path, it was able to make the natural frequency independent of amplitude. This theory is discussed below. In effect, the pendulum length decreased as the magnitude of its swing angle increased, and this altered its natural frequency. However, that made little difference to the irregularity of the pendulum torque, Fig. 3(a), because the radius vector from the centre of rotation to the pendulum mass was only slightly altered by a change in pendulum length. For roller pendulums, the same limitation applied.

\section{VARIABLE LENGTH PENDULUMS}

The concept of a bifilar pendulum whose natural frequency is independent of its amplitude was introduced by Denman in 1992. ${ }^{4}$ Denman showed that, if the path of the centre-of-mass is described by an epicycloidal curve, its natural frequency is constant. 


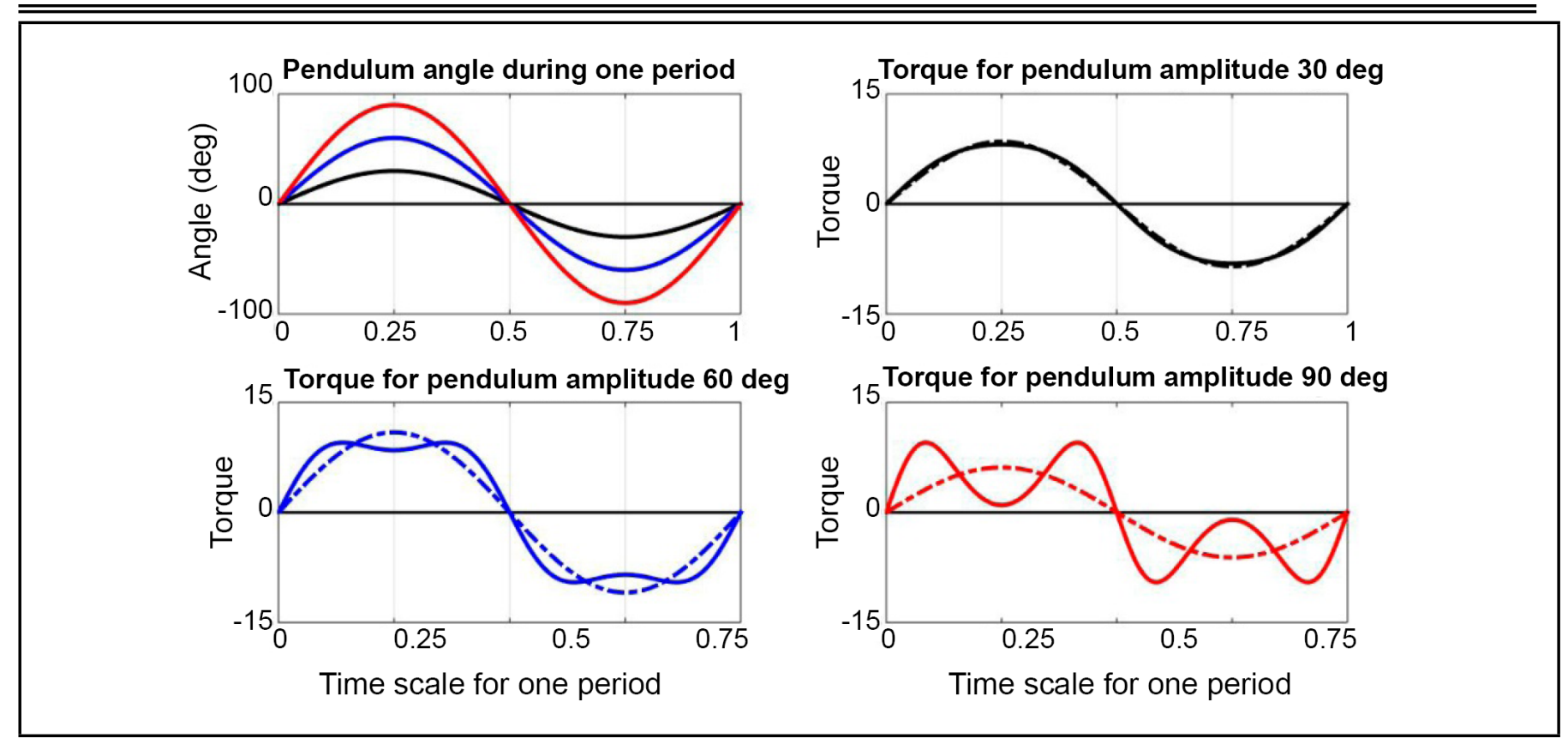

Figure 3. Time history of non-dimensional pendulum torque $T / \mathrm{mal}^{2}$ during one full period of sinusoidal pendulum motion at three different amplitudesof $30^{\circ}$ (upper right), $60^{\circ}$ (lower left) and $90^{\circ}$ (lower right). The time history of pendulum displacement is shown upper left. These diagrams are for $n=4$ and $\epsilon=0.1$, but those parameters are not critical. These results are for a bifilar pendulum following a circular path, but those for both a tautochronic pendulum and a roller pendulum are very similar.

An approximate analysis (see Appendix 2) showed that the free motion of a pendulum will be simple harmonic with constant natural frequency if its length $l(\phi)$ changed with swing angle $\phi$ according to the sinc equation $l(\phi)=L \sin \phi / \phi$.

Figure 4(a) compares the trajectories of a pendulum mass for a circular trajectory in red (a traditional constant length pendulum), sinc trajectory in blue and epicycloidal trajectory in green. The last was generated by the small green circle rolling on the large green circle. All the trajectories were for $n=4$ when the radius from the centre of rotation to the centre of the pendulum is $n^{2}=16$ times the length of the (simple) pendulum. The centre of rotation of the carrier wheel was at $O$ and the (simple) pendulum was hinged to its carrier at $A$.

Figur 4(b) is a magnified view of the alternative trajectories. The dimensions shown and position of the bump stops are for a typical automobile application to illustrate the small differences between the three trajectories.

\section{STEADY HARMONIC CALCULATIONS}

Theoretical calculations of the effectiveness of a particular installation are usually made by the application of some method of harmonic balance in which the motion of the pendulum is approximated by an assumption of harmonic time dependence, responding to the chosen harmonic of engine torque. This method was explored in the author's original studies and has been used in various forms extensively since. ${ }^{9,10}$ Harmonic balance calculations consider the rotational equilibrium of the assembly of a carrier wheel and pendulum and allow the relationship between pendulum displacement amplitude, carrier displacement amplitude and pendulum torque amplitude to the found with good accuracy.

Typical results are shown below, for a constant length bifilar pendulum, Figs. 5(a), 5(c), and a roller pendulum, Figs. 5(b), 5(d), following a circular track. Results are shown for different values of detuning, $\epsilon=0.05,0.1,0.15,0.2,0.25$. In 5(a) and 5(b), the amplitude of (non-dimensional) pendulum torque was plotted against pendulum amplitude, in 5(c) and 5(d), pendulum torque was plotted versus carrier amplitude. The assumption was that the excitation was purely harmonic and that the corresponding harmonic of pendulum amplitude and carrier amplitude was identified by a harmonic balance calculation. For the results here, a version of the Ritz Minimising Method has been used, but other methods produce similar results. ${ }^{9}$ The essential result was that, as pendulum amplitude increased, the carrier reaction torque increased until it reached a maximum, but thereafter decreased. The corresponding carrier amplitude at first increased (for $\epsilon>0$ ) with increasing torque amplitude, but then reduced to zero at an "optimum" condition, before changing phase and approaching a jump instability.

Figure 5 shows non-dimensional torque $T / m \Omega^{2} a l$ as the ordinate. To compare the magnitude of the torques generated by bifilar and roller pendulums, an allowance must be made that roller inertia reduces a roller's natural frequency so that dimension $l$ is different in the two cases. Therefore, in the later Fig. 7, the ordinate is changed to $T / m \Omega^{2} a^{2}$ to allow a direct comparison between bifilar and roller pendulums. Also, as already pointed out, since a bifilar construction fits within the crankcase of an engine, it can usually be larger and heavier than a wheel-mounted roller pendulum.

These results were for a conventional pendulum or roller following a circular trajectory. It was assumed that the roller does not slip or partially slip. There was some evidence that this may sometimes happen. If it did, the effective inertia of the roller was reduced.

When their trajectory departed from circular, the results were modified. Figures 6(a), 6(b) show carrier amplitude plotted against pendulum amplitude for both circular and noncircular (constant-frequency) trajectories. As in Figs. 5, there was a separate curve for each value of detuning, in this case for $\epsilon=0.0,0.05,0.1,0.15,0.2,0.25$.

For both Figs. 6(a) and 6(b), the solid lines are for a circular pendulum trajectory, the broken lines for a sinc (constant frequency) trajectory. To achieve zero carrier amplitude for a constant length pendulum, the value of detuning must be chosen to 


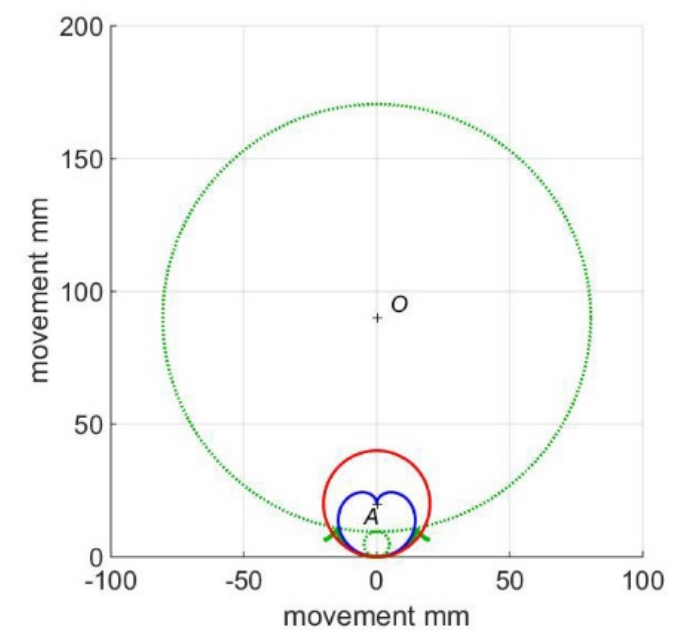

(a)

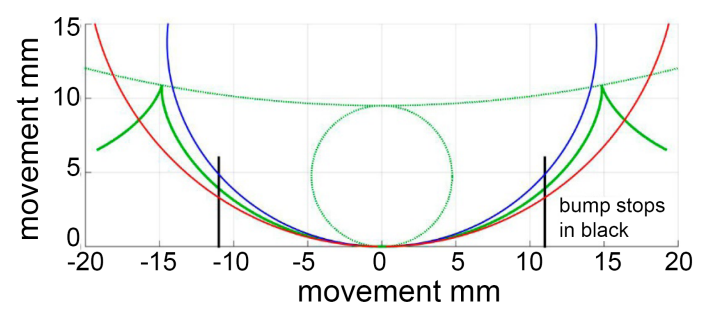

(b)

Figure 4. (a) Illustrating the generation of an epicycloid trajectory (in green), circular trajectory (in red) and sinc trajectory (in blue). The larger green circle's centre is the centre of rotation of the carrier wheel. The pendulum pivots about A. Magnified detail is shown in Fig 4(b). Dimensions shown are for a typical automobile application. (b) Part-view of 4(a). The red trajectory is circular for a constant length pendulum. Blue and green trajectories are for the centre of mass of a pendulum of varying length. The blue trajectory is a sinc curve generated by equation (B.4). The green trajectory is an epicycloidal curve with proportions so that it has the same radius of curvature as the red and blue curves when in their central position $x=y=0$. The position of bump stops in a typical application is where shown.

suit the intended pendulum amplitude, while for a tautochronic pendulum, detuning should ideally be zero. This gave a tautochronic pendulum an advantage, although in practical terms it may be difficult to realise this (see below).

As already noted, when excitation generated an excessive pendulum torque for which there was not a stable harmonic solution, jumping occurred and the pendulum became unstable and buffets between its stops.

Critical values of pendulum harmonic torque amplitude are shown in Figs. 7(a) and 7(b). Six different cases are shown in each figure. Blue curves are for a constant length pendulum; red curves for a sinc (constant frequency) pendulum, green curves are for a roller pendulum. In Fig. 7(a), results are shown for orders $n=2$ and $n=4$ and for different values of detuning $\epsilon$ from 0 to 0.25 in 5 equal steps. In Fig. 7(b), the corresponding results are shown for $n=0.5$ and $n=1$. For both Figs. 7, non-dimensional pendulum torque is defined as $T / m a^{2} \Omega^{2}$ instead of $T / m a l \Omega^{2}$.

The conclusion from the design diagrams, Figs. 7, is that, while a tautochronic (constant frequency) design made it easier to ensure zero or very small carrier amplitude, this was sub-

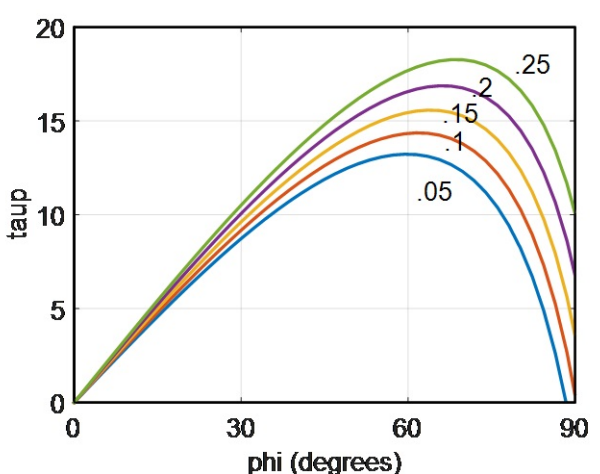

(a)

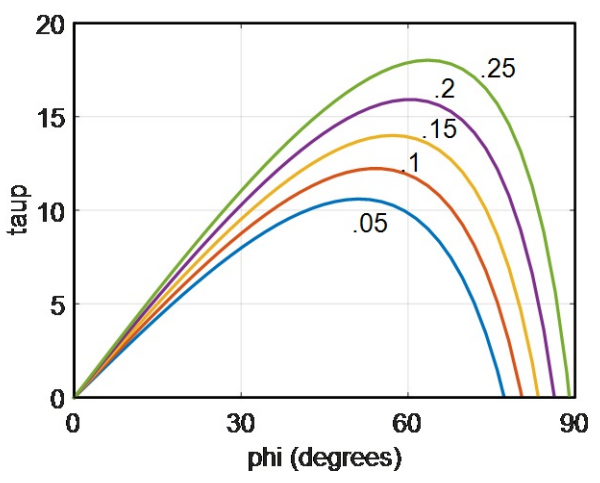

(b)

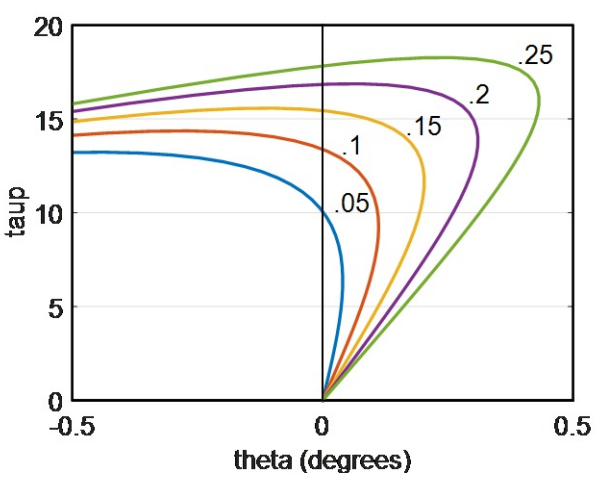

(c)

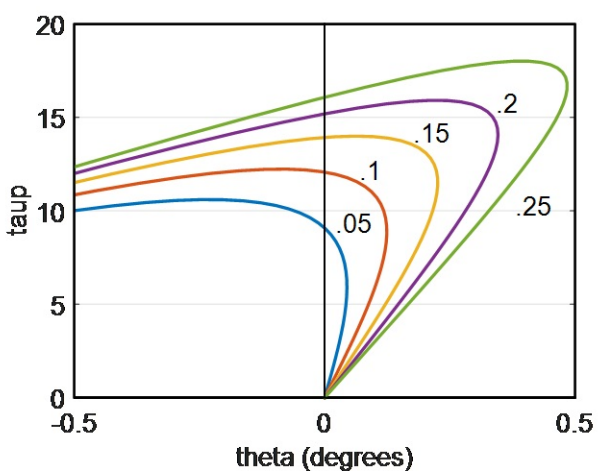

(d)

Figure 5. Approximate harmonic response amplitudes for a bifilar and a roller pendulum $\left(I / m r^{2}=0.5, r / l=4\right)$, both with circular trajectories, that have synchronised with the $n=$ 4 order of engine excitation for different values of detuning $\epsilon$. In these figures, the non-dimensional pendulum torque is defined as taup $=T /$ mal $\Omega^{2}$, phi $=\phi$ is pendulum swing angle, thet $a=\theta$ is carrier vibration amplitude (superimposed on its steady angular velocity). 


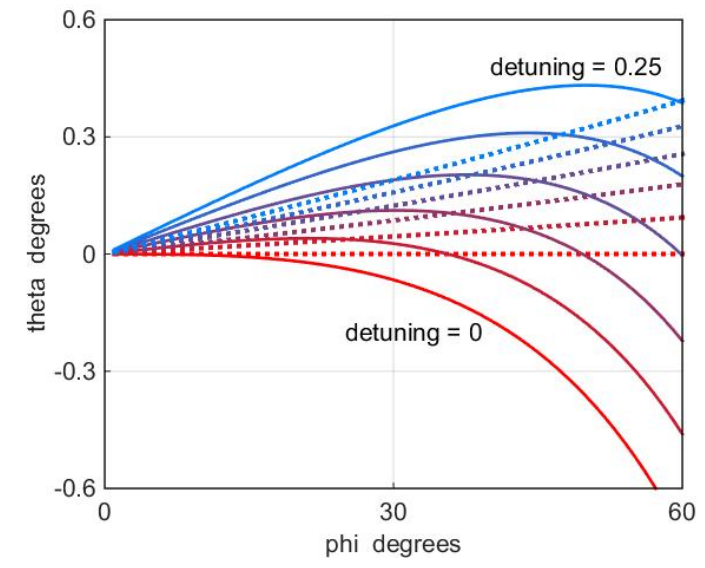

(a)

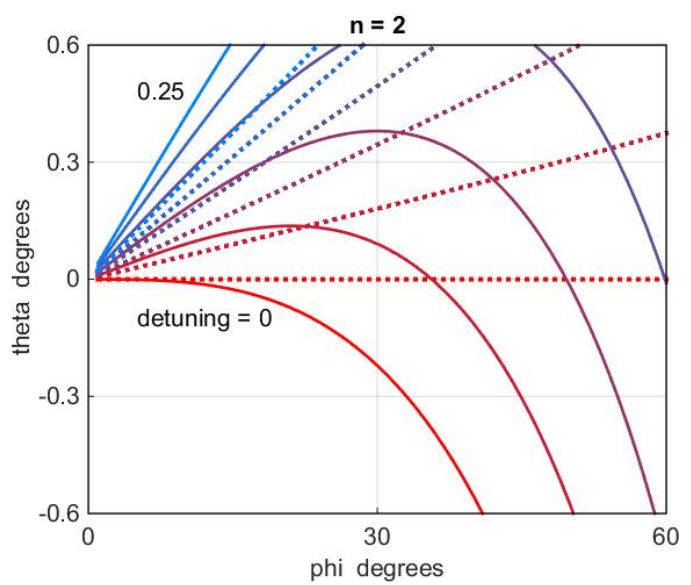

(b)

Figure 6. (a) Carrier amplitude versus pendulum amplitude for 6 values of detuning and $n=4$. Solid lines for a circular trajectory; broken lines for a sinc (constant frequency) trajectory. (b) The same as Fig 6(a) except that $n=2$.

ject to the maximum pendulum torque amplitude not being exceeded. These calculations indicated that a tautochronic pendulum reached its peak harmonic torque at a (slightly) lower swing amplitude than a corresponding pendulum of constant length.

All harmonic calculations, of which these are examples, deal only with the calculated fundamental component of pendulum torque. As can be seen from Figs 4, when pendulum amplitude approaches $60^{\circ}$ this involves a substantial approximation as the amplitude of the next, $3^{\text {rd }}$, harmonic approaches the amplitude of the fundamental.

\section{PENDULUMS ON THE SAME WHEEL}

A further complication occurs when pendulums are attached at several places in a rotating system that has many separated vibratory torque inputs, such as a multi-cylinder reciprocating engine. Then the torque which one pendulum may be called on to resist may arise from the vector sum of some or all of the excitation sources, modified by the dynamic torsional response of the connecting assembly. Alternatively, many pendulums may be mounted on the same carrier wheel. This is the usual construction for automobile engines, when several centrifugal pendulums are mounted on one wheel (see Figs. 1(b), 2(b), and 2(d)). It is important that all these individual pendulums play an equal part, rather than one or two generating most of the reaction torque and therefore over-swinging. It has been

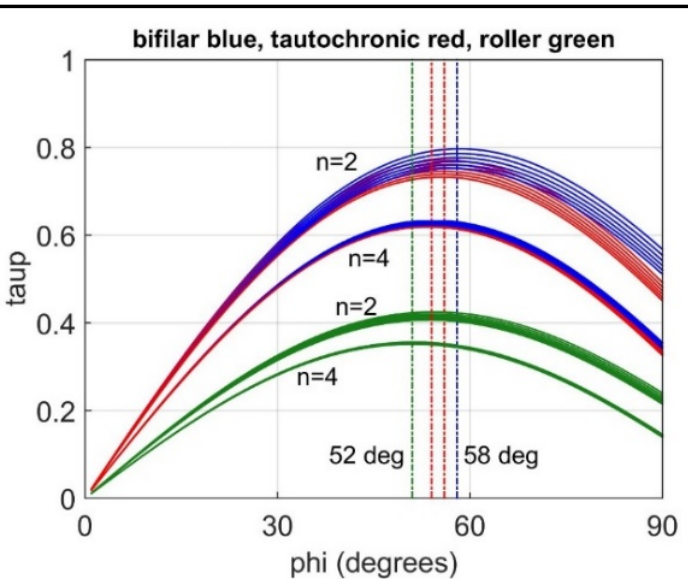

(a)

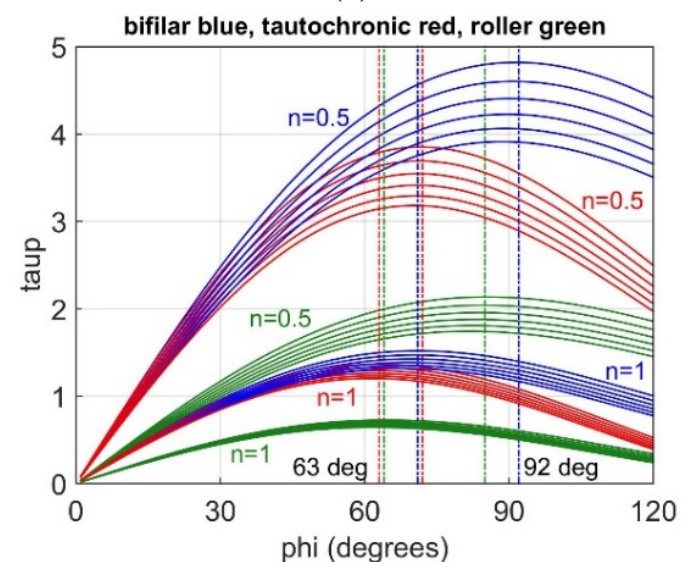

(b)

Figure 7. 7.(a) and (b): Amplitude of harmonic pendulum torque (taup $=T / m a^{2} \Omega^{2}$ ) plotted against pendulum amplitude ( $\mathrm{phi}=\phi)$ for $n=2$ and $n=4$, Fig. 7(a), and for $n=0.5$, $n=1$, Fig. 7(b). The blue curves are for a constant length bifilar pendulum, the red curves for a sinc (tautochronic) pendulum, the green curves for a roller pendulum in a circular track. Detuning increases from $\epsilon=0$ to $\epsilon=0.25$ in 5 steps going from below to above. For the roller pendulum, $I / m r^{2}=1 / 2$, $r / a=1 / 5$. The values of $\phi$ for which there is a potential nonlinear jump lie between $52^{\circ}-58^{\circ}(n=2,4)$ and $63^{\circ}-92^{\circ}$ $(n=1 / 2,1)$ with the roller pendulum jumping slightly before the tautochronic pendulum and the constant length pendulum.

found (see e.g. Shaw \& Geist, 2010) that, in the theoretical case when all of several rollers are tuned to precisely the same order with no detuning, energy may be shared unequally between the separate rollers. ${ }^{12}$ By intentionally slightly detuning each of several multiple pendulums, whether these are constant length or tautochronic pendulums, it has been found that significantly uneven localised responses can be avoided.

\section{SYSTEM CALCULATIONS}

Relatively little attention has been given to the analysis of multi-pendulum configurations, when crankshaft-mounted centrifugal pendulums are combined with wheel-mounted pendulums on an engine's flywheel. Establishing an accurate model for the torsional vibration response of a system without absorbers, and then introducing multiple centrifugal pendulums, which are essentially nonlinear devices, brings formidable computational problems. But interesting results are found.

Figures $8(a)$ and 8 (b) show results for a simple two degree- 
of-freedom torsional system, with one pendulum on the wheel that carries $4^{\text {th }}$ order excitation. The horizontal axis in Fig. 8(a) represents frequency, expressed as the ratio of the speed of rotation to the speed at which the $4^{\text {th }}$ order of $\Omega$ equals the torsional natural frequency of the system without its pendulum, $\Omega_{c}$.

Figure 8(a) shows loci of carrier amplitude plotted against frequency for different amplitudes of applied harmonic torque. Loci were plotted for four different excitation torque amplitudes $T / m \Omega^{2} a l$ (which are constant): 10 (blue), 13.4 (red) 15 (green) and 25 (black). The system parameters were chosen so that the second wheel acted as a vibration absorber for the first wheel when $\Omega=0.5 \Omega_{c}$. For this example, $\epsilon=0.06$.

Figure 8 (b) goes further, for the same system. The top graph in Fig. 8(b) is similar to 8(a) with different contours; the middle graph shows pendulum swing amplitude against frequency, the bottom graph pendulum torque amplitude against frequency. In Fig. 8(b), loci were plotted for four different excitation torque amplitudes (which are constant): 5 (red), 10 (green), 13.2 (black) 13.6 (blue) and 25 (magenta). When the torque amplitude was 13.6, a nonlinear instability occurred when $\Omega / \Omega_{c}$ approached 1 , the system's critical speed. For torque of 13.2, this instability was just avoided.

An interesting feature arose for speeds close to $\Omega / \Omega_{c}=0.5$. Additional instabilities occurred. A much-magnified detail of the top graph in Fig. 8 is shown in Fig. 9. This looping of the loci was a curious complication, with one side of each loop describing an unstable solution from the harmonic balance calculation. The unstable solutions did not occur in practice, the operating point finding its nearest stable position. This behaviour, computed by an analogue simulation, is shown in the inset view. ${ }^{9,11}$

Calculations for multi-degree-of-freedom systems have become increasingly complicated. The results from an early unpublished conference contribution by the author are shown in Fig $10 .{ }^{11}$ The response of a system with three wheels, two with (constant length) pendulums attached, each with $\epsilon=0.0625$, and each subjected to $4^{\text {th }}$ order in-phase harmonic torque excitation as shown (Fig. 10(a)) are plotted. In Fig. 10(a), results are shown for six different frequencies (expressed as a speed ratio by $\frac{\Omega}{\Omega_{c}}$ where $\Omega$ was the lower non-zero natural frequency of the system). In Fig. 10(b), the response for continuing increase in torque amplitude is shown for the single case $\frac{\Omega}{\Omega_{c}}=1$. As excitation exceeded the first jump value, the response moved to the next stable position. Pendulum 1 followed the marked sequence $\mathrm{A}_{1}, \mathrm{~B}_{1}, \mathrm{C}_{1}, \mathrm{D}_{1}, \mathrm{E}_{1}$ and pendulum 2 , the sequence $A_{2}, B_{2}, C_{2}, D_{2}, E_{2}$. This diagram assumed that the pendulums had freedom to swing through the large angle required without meeting any restraints, which in practice will not necessarily be the case. The consequence of pendulums repeatedly hitting their stops is discussed in section 10 .

Now that centrifugal pendulum absorbers are increasingly used in automobile engines, and as designs combining crankshaft-mounted with flywheel-mounted pendulums become more common, research to study operation under transient conditions is needed to explore the disruptive effect of engine acceleration, and to widen the speed range over which satisfactory vibration absorption can be achieved.

\section{MEASUREMENTS}

The results of measurements on a typical automobile centrifugal vibration absorber show that the trajectory of the centre-of-mass follows is circular to close accuracy. To convert this to a tautochronic path, the tautochronic path would deviate by less than $1 \mathrm{~mm}$ from a circular path even if the pendulum's amplitude was as much as $60^{\circ}$. Measurements were made by a camera fitted with a multi-exposure shutter focussed on the sharp corner of a pendulum, Fig. 11(a).

By only moving the pendulum, while shooting multiple images, Fig. 11(b), its trajectory could be identified accurately and compared with an exactly circular trajectory, Fig. 11(c). The divergence from a perfect circle was extremely small and appeared to be within the limits of manufacturing accuracy. This complication of providing a wear-resistant non-circular track to the accuracy required for a tautochronic path mitigated against the adoption of a tautochronic design.

\section{DESIGN}

There were two principal design considerations. The pendulums had to be correctly tuned. Their maximum permissible torque must not be exceeded.

The first involved selecting dimensions to satisfy the natural frequency equations (A1.3) for a bifilar pendulum, (A1.6) for a roller pendulum. Provided that the bifilar pendulum was suspended so that it always moved parallel to its carrier wheel, with no relative rotation, the pendulum inertia and mass did not enter into (A1.3). In the case of a roller pendulum, the ratio $I / m r^{2}$ did affect the natural frequency, with frequency decreasing as roller inertia increased.

The maximum permissible torque can be found from Fig. 7. This enabled the permitted pendulum amplitude and torque to be read from the diagram. Although Fig. 7 has been calculated from applying equations (A2.1), (A2.6) and (A2.8), these calculations had to be done numerically and the fundamental component of pendulum torque computed to ascertain its magnitude. The calculations that led to the graphs in Fig. 7 can be repeated, but the graphical presentation shown there has sufficient accuracy for most practical applications.

The need for these two design parameters to be calculated was discussed by Mitchiner and Leonard, but they did not consider bifilar and roller pendulums, only a pendulum swinging from a single point of suspension for reducing the torsional vibration of an air compressor. ${ }^{8}$ Also their paper did not consider pendulum dynamics outside the linear range, which was suggested should be limited to a pendulum amplitude of $0.1 \mathrm{rad}$ $\left(5.7^{\circ}\right)$ or $0.2 \operatorname{rad}\left(11.4^{\circ}\right)$ as an absolute maximum.

As will be seen from Fig. 7, significantly higher swing amplitude was permissible before instability was approached. Even with a safety factor of 2, the maximum permissible pendulum amplitude (for all three types of pendulum) may be as high as $26^{\circ}(0.45 \mathrm{rad})$.

When excitation was distributed along a torsionally-flexible drive shaft and pendulum absorbers could not be mounted directly at the points of excitation, their dynamics were affected by torsional vibration of the flexible assembly. A general analysis of such a system has not been completed so far as this author is aware. Examples of the recent analysis by the author of torsional systems with limited complication are shown in Figs. 8 and 9 which are new.

There was considerable skill in designing a satisfactory absorber that would provide enough vibration absorption and would last the working life of an engine. Both the design and the analysis of these devices still bring formidable challenges and there are numerous practical considerations.

Strength is one. Very high g forces are generated on the pendulums, which may be as high as $1000 \mathrm{~g}$, and creep of the support structure under these loads may occur during long service. 


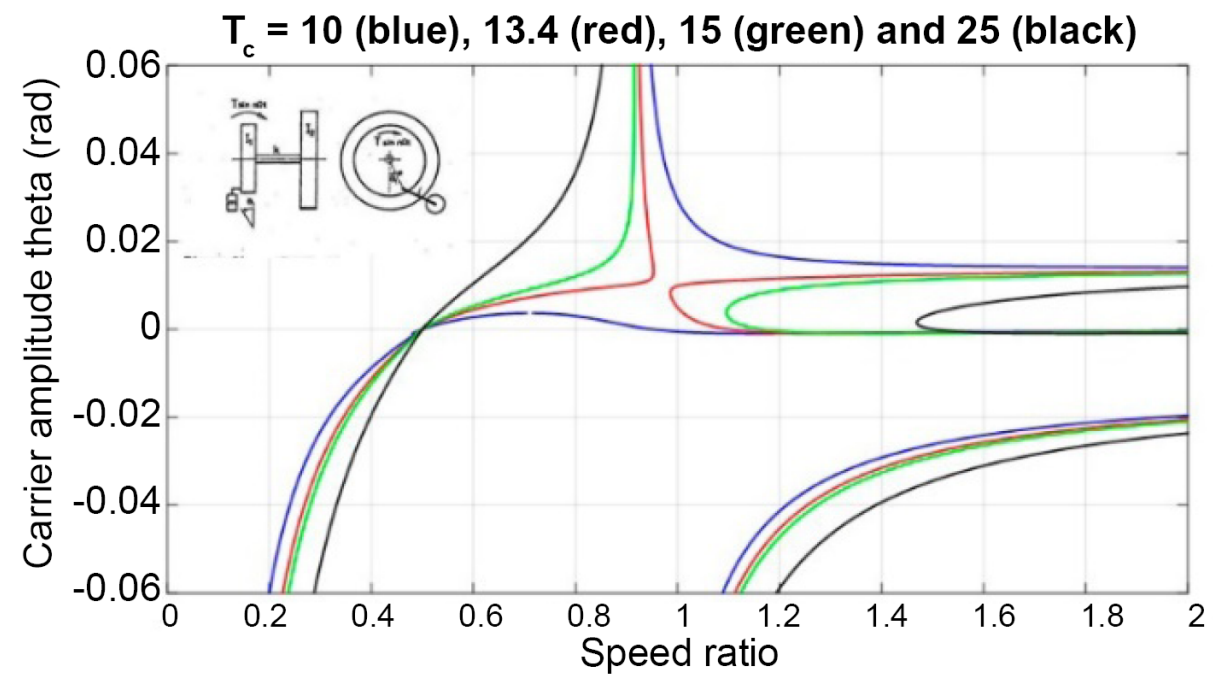

(a)
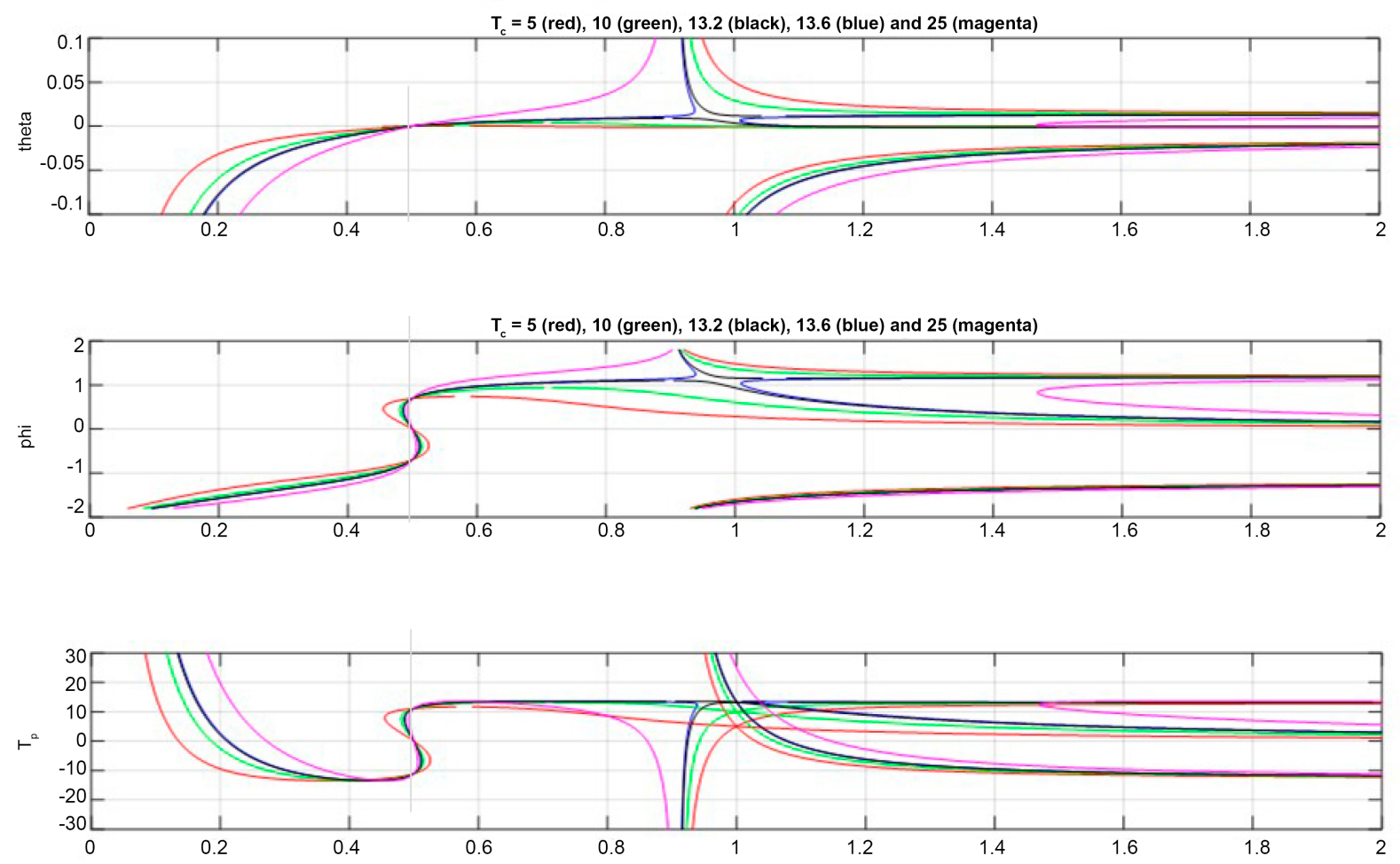

(b)

Figure 8. (a) Approximate harmonic response of a torsional system with two wheels, with excitation on the wheel carrying a pendulum (see inset, top left). Carrier wheel amplitude is plotted against speed. The speed ratio is 1.0 when the excitation frequency coincides with the natural frequency of the system. It is 0.5 when the right-hand wheel acts as a perfect vibration absorber for the left hand (carrier) wheel. Each graph is for a different value of the non-dimensional excitation amplitude. (b)The same system as Fig 8(a). The top figure shows carrier amplitude against excitation frequency, the middle graph pendulum amplitude, the bottom graph pendulum reaction torque amplitude on the carrier wheel. Note that the colour coding for Figs 8(a) and $8(\mathrm{~b})$ is different.

Lubrication was important under the highly-stressed rollingcontact conditions. For crankshaft-mounted bifilar pendulums, this may not be a problem because of the crankshaft lubrication system, but roller pendulums need a low-viscosity highpressure lubricant to be sealed within each roller's housing; otherwise surface pitting may occur due to surface fatigue under high contact stresses. The roller surface may have to be circumferentially grooved to provide a lubrication pathway.
To reduce wear, further complications may include the introduction of shrink-fit liners, as shown in Fig. 1(b). And to increase the magnitude of the reaction torque generated by a pendulum, tungsten $(\rho=19.5)$ may replace stainless steel $(\rho=8)$.

As explained above, as an engine speeds up, at first its loose pendulum weights rattle, when there is insufficient centrifugal force to pull them into synchronism. Sometimes they can 


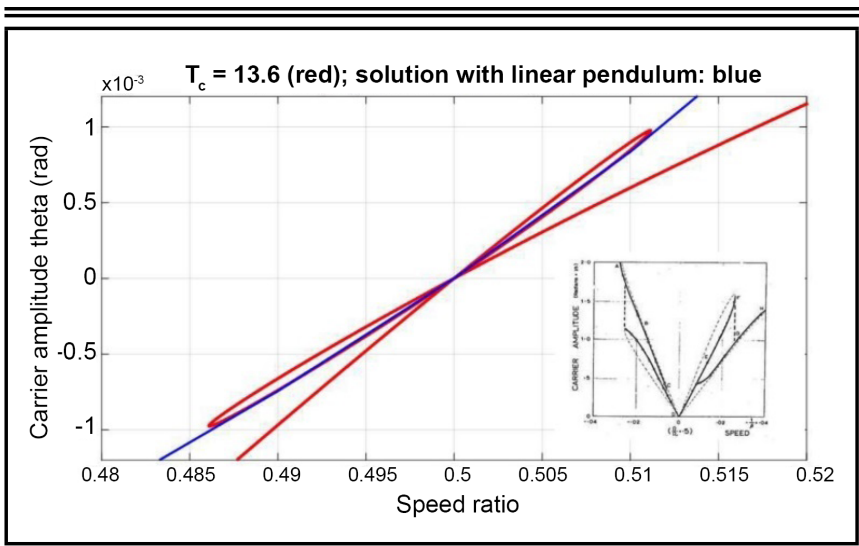

Figure 9. Enlarged view of carrier amplitude against speed around the speed at which the second wheel acts as a vibration absorber for the first wheel.

be heard audibly falling into synchronism, when there was a change in the noise emitted and engine smoothness improved. This behaviour has long been known to operators of aircraft with reciprocating engines. A 1976 Operator's Manual, approved by the FAA, for aircraft engines with centrifugal pendulum vibration absorbers, carried this cautionary warning: "These engines are equipped with a dynamic counterweight system and must be operated accordingly,....Use a smooth, steady movement of the throttle (avoid rapid opening and closing). If this warning is not heeded, there could be severe damage to the counterweights, roller and bushings."

Modern automobile engine designs incorporate a margin of strength intended to be sufficient to absorb the hammering that occurs when there are frequent sudden stops and starts.

\section{CONCLUSIONS}

The long-term future of internal combustion engines burning fossil fuels is now an issue. Eventually, high-performance electric drives are likely to supersede most reciprocating automobile power plants. However, simple long-stroke, low emission gasoline engines, perhaps supplementing electric traction, may find their place. Any reciprocating engine has a tendency to generate torsional vibration and lightweight, and singlecylinder engines are no exception. They are still likely to incorporate centrifugal pendulum vibration absorbers.

\section{ACKNOWLEDGEMENTS}

The author is grateful for information from, discussions with, and, where requested, permission to use illustrations provided by Engineered Propulsion Systems Inc. www.eps.aero; Lycoming Division of the Avco Corporation www.lycoming.textron.com; Schaeffler Technologies GmbH \& Co. KG www.schaeffler.de; TCI Automotive www.tciauto.com; and Vibration Free Ltd www.vibrationfree.co.uk .

\section{A. APPENDIX 1}

Linear theory of bifilar and roller centrifugal pendulums Nomenclature

The following definitions will be adopted. Dimensions are illustrated in Fig. A.1.

For a bifilar pendulum, Fig. A.1(a), with centre of rotation at $O$, centre of mass at $G$, and pendulum links of length $A C$ :
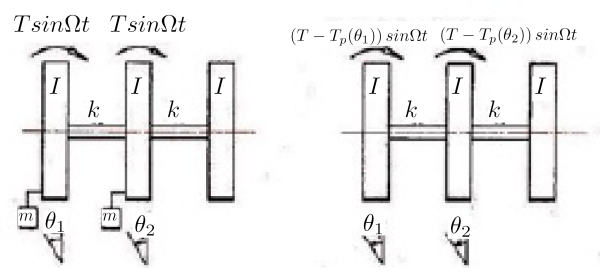

Multi-component system with pendulums positioned at axis excitation source.
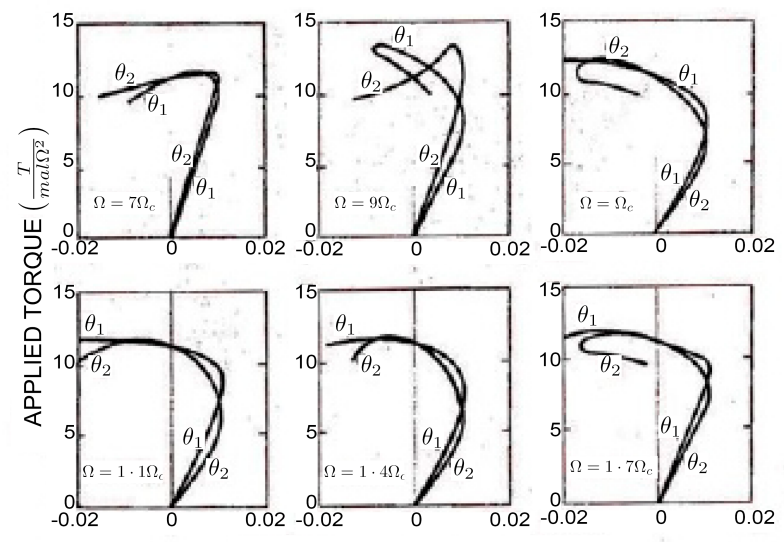

CARRIER AMPLITUDE (RADIANS)

(a)

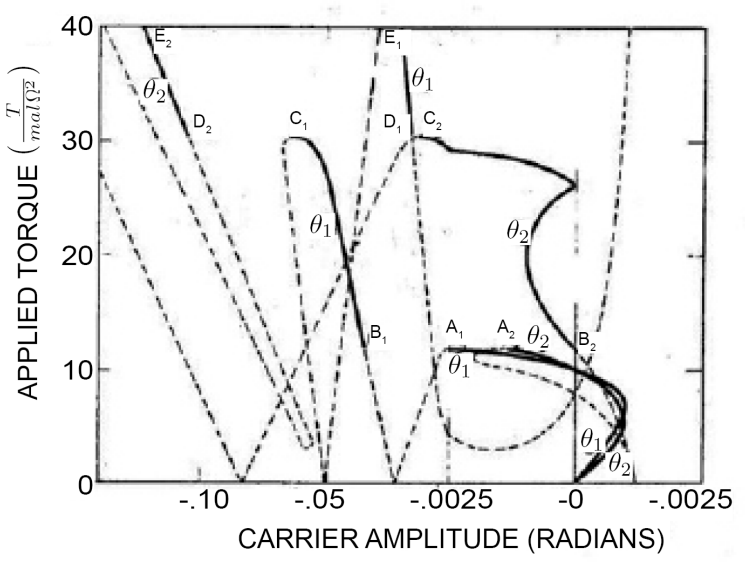

(b)

Figure 10. (a) Response of the two carrier wheels in the system shown above to increasing (in-phase) torque at different rotational speeds from $0.7 \Omega_{c}$ to $1.7 \Omega_{c}$ for $n=4$ and $\epsilon=.0625$ for both pendulums. ${ }^{911}$ (b) Response of both carrier wheels for the system of Figure 10(a) for harmonic excitation torque amplitude of greater magnitude at speed $\Omega_{c}$, the lower (nonzero) natural frequency of the system. ${ }^{9,11}$

$d_{1} \quad$ Aperture diameter in pendulum and carrier (not shown)

$d_{2} \quad$ Diameter of cylindrical roller joining pendulum to carrier (also not shown)

$l \quad d_{1}-d_{2}=$ pendulum's length $A C$

(the length of each arm of a bifilar suspension)

a Radial distance $O A+C G$ from the wheel's centre of rotation to the centre of mass of pendulum (in its undeflected position, excluding pendulum length)

$m$ Mass of pendulum

$I \quad$ Inertia of pendulum about its centre of mass, $G$

For a roller pendulum, Fig. A.1(b), with centre of rotation 


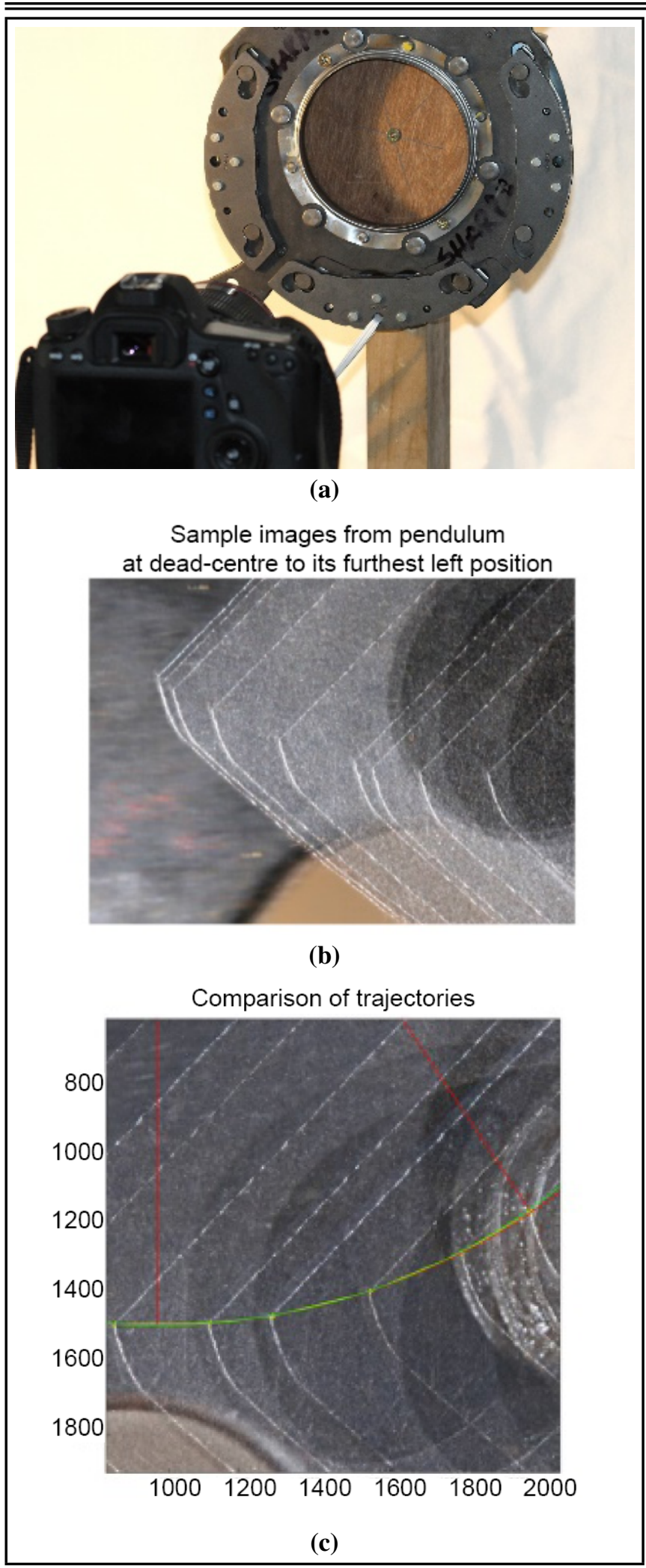

Figure 11. (a) Experiment to record the pendulum trajectory for a typical automobile bifilar centrifugal pendulum. (b) Multi-exposure recording the trajectory of the bifilar pendulum in Fig. 11(a). (c) Best-fit trajectory (green) compared with circular trajectory (red) and theoretical tautochronic trajectory (yellow) for the bifilar pendulum in Fig 11(b).

at $O$, circular aperture with centre at $A$, and roller with centre $C: \quad$ Radius of aperture within which roller is constrained, dimension $A P$

$r \quad$ Radius of roller, dimension $C P$

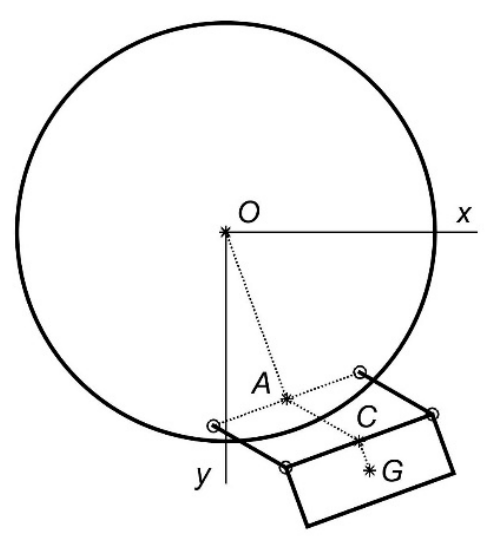

(a)

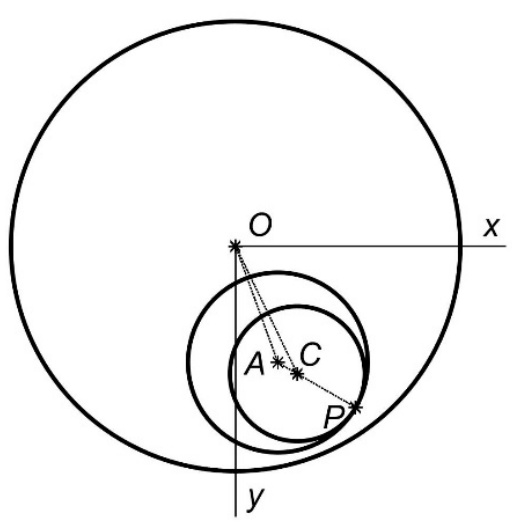

(b)

Figure A.1. (a) Geometry of a bifilar centrifugal pendulum. (b) Geometry of a roller centrifugal pendulum.

$l$ Difference in radii, $R-r$ dimension $A C$

a Radial distance from the centre of rotation to the centre of aperture, dimension $O A$

$m$ Mass of roller $I$ Inertia of roller about its centre of mass $C$

For both pendulums:

$\Omega \quad$ Average rotational speed of carrier wheel ( $\mathrm{rad} / \mathrm{s})$

$n \quad$ Order of vibration to which the pendulum is tuned (pendulum frequency tuned to $n$ times $\Omega$ )

$\Omega t+\theta \quad$ Angle made by $O A$ with the fixed axis $O y$, $t=$ time $(\mathrm{s})$

$\phi \quad$ Pendulum swing angle relative to the carrier wheel which is the (acute) angle made by $A C$ with $O A$ extended

The bifilar pendulum's natural frequency for smallamplitude (linear) vibrations at rotational speed $\Omega$ was

$$
\omega_{0}=\Omega \sqrt{a / l} ;
$$

so that to absorb a harmonic of frequency $n \Omega$,

$$
n^{2}=a / l
$$

To allow for the pendulum frequency $\omega_{0}$ decreasing slightly as the pendulum amplitude increased (assuming the pendulum center of mass followed a circular arc), in practice $a / l$ was set slightly more than $n^{2}$ according to:

$$
n^{2}(1+\epsilon)=\frac{a}{l}
$$

where $\epsilon$ was defined as the detuning. 
On substituting for $a / l$ from (A.3), the inertia added by a bifilar pendulum may be expressed as:

$$
I+m(a+l)^{2}+\frac{m(a+l)^{2}}{\epsilon}
$$

which was an alternative form of: ${ }^{3}$

$$
I+\frac{m a(a+l)^{2}}{\left(a-l n^{2}\right)} .
$$

In the case of a roller pendulum, the analysis was more complicated. For the definitions given above, the natural frequency of a roller in its track was:

$$
\omega_{0}=\Omega \sqrt{a / l} \frac{1}{\sqrt{1+I / m r^{2}}}
$$

so that the order of vibration for resonance was:

$$
n^{2}=\left(\frac{a}{l}\right) \frac{1}{\left(1+\frac{I}{m r^{2}}\right)} ;
$$

and detuning $\epsilon$ is defined so that:

$$
n^{2}(1+\epsilon)=\left(\frac{a}{l}\right) \frac{1}{\left(1+\frac{I}{m r^{2}}\right)} .
$$

With these definitions, the effective inertia offered by one roller was:

$$
I+m(a+l)^{2}+\frac{m(a+l-I / m r)^{2}}{\epsilon\left(1+\frac{I}{m r^{2}}\right)}
$$

corresponding to (A.4) for the bifilar pendulum.

Derivation of (A.9) followed from the equations of motion for small-amplitude movement of the roller when its carrier wheel was subjected to forced harmonic excitation and assumed that there was no slipping.

\section{B. APPENDIX 2}

\section{Nonlinearity of a centrifugal pendulum \\ Bifilar pendulum with constant length links}

Consider the model shown in Fig B.1. Arm OA rotates about the fixed centre $O$ at angular velocity $\Omega$. A simple pendulum of point mass $m$ and length $l$ was hinged at $A$.

Assume a quasi-static analysis, when the only force on the pendulum mass came from the centrifugal acceleration $\Omega^{2} R$ where $R$ was the distance from $O$ to $C$, the centre of mass.

This centrifugal force generated a tension in the pendulum arm, which applied a load at $A$ and exerts a torque about $O$. The component of centrifugal force perpendicular to the pendulum caused the pendulum to rotate but did not apply a load at its point of support $A$. The analysis was made slightly more complicated when the additional centrifugal acceleration arising from the pendulum's relative angular velocity $d / d t(\phi)$ was included, but the result was the same in principle.

The outwards centrifugal force on the pendulum mass $m$ was $m R \Omega^{2}$ where $R$ was the length $O C$. The torque about $O$ exerted by this force was $T=p m R \Omega^{2} \cos \theta$ where $p$ was the moment arm shown in Fig. B.1. By geometry, if the pendulum swung through angle $\phi$ relative to the wheel, $p=a \sin \phi$ and $R \cos \theta=(l+a \cos \phi)$, so that:

$$
\frac{T}{m a l \Omega^{2}}=\left(1+\left(\frac{a}{l}\right) \cos \phi\right) \sin \phi .
$$

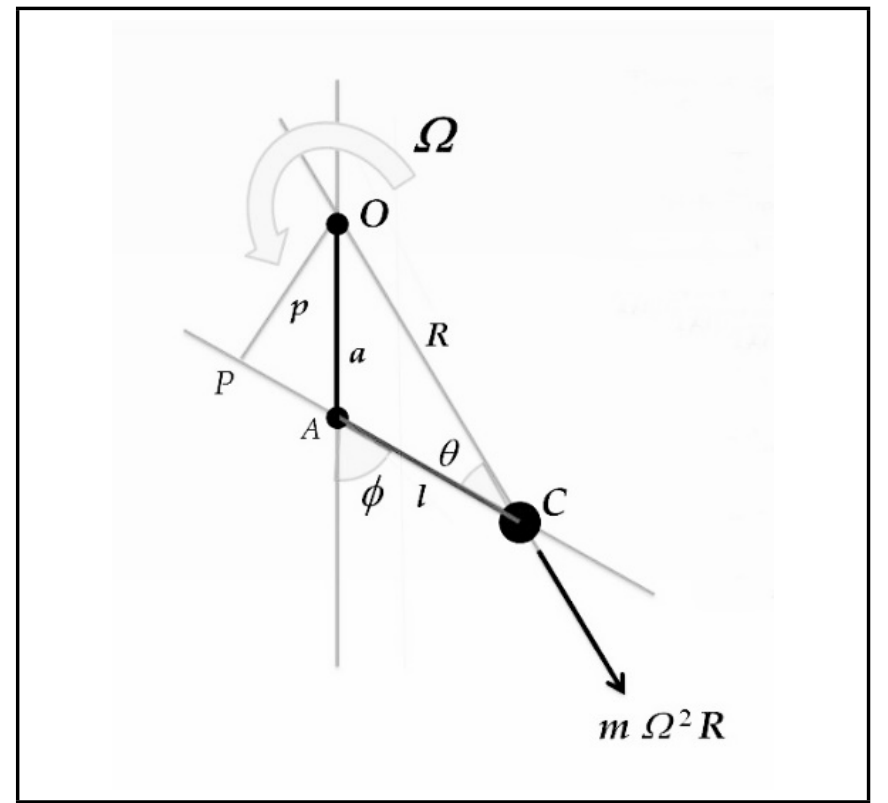

Figure B.1. Simplified quasi-static analysis of a centrifugal pendulum. Arm $O A$ rotates about the fixed centre $O$ at angular velocity $\Omega$. A simple pendulum of point mass $m$ and length $l$ is hinged at $A$.

The results of such a calculation are shown in Fig. 3(a). The solid line shows the time-history of non-dimensional pendulum torque $T / \mathrm{mal}^{2}$ during one full period for sinusoidal pendulum motion at an amplitude of $60^{\circ}$. The fundamental component of this response is shown by the broken line. It can be seen that, during each period of the pendulum's motion, the torque it exerted on its carrier wheel did not change harmonically, but instead followed the irregular curve shown. For this example, the order $n=4$ and the detuning zero, but the irregular torque was not sensitive to these values.

\section{Bifilar pendulum with variable length links}

Because the natural period of a centrifugal pendulum increased with amplitude, so that its natural frequency decreased, much has been written about reducing the effective length of the pendulum to achieve a constant frequency pendulum, or so-called tautochronic pendulum. ${ }^{10,11}$

For a simple pendulum attached to a carrier wheel, which rotated at constant angular velocity $\Omega$, Fig. B.1, the pendulum equation was:

$$
m l \ddot{\theta}+m R \Omega^{2} \sin \theta=0 ;
$$

and, since $R \sin \theta=a \sin \phi$,

$$
\ddot{\phi}+\Omega^{2}\left(\frac{a}{l}\right) \sin \phi=0 .
$$

Therefore, provided that the pendulum length $l$ changed, according to:

$$
l=L \sin \phi / \phi
$$

motion was simple harmonic motion with constant frequency $\Omega \sqrt{a / L}$. Second-order terms from the Coriolis acceleration arising from changing $l$ were neglected.

For this case, equation (B.1) became:

$$
\frac{T}{m a L \Omega^{2}}=\left(l / L+\left(\frac{a}{L}\right) \cos \phi\right) \sin \phi ;
$$

where $L$ was the pendulum length when at its centre and, substituting from (B.4),

$$
\frac{T}{m a L \Omega^{2}}=\left(\sin \phi / \phi+\left(\frac{a}{L}\right) \cos \phi\right) \sin \phi .
$$


A graph of $T / m a L \Omega^{2}$ from has comparable nonlinearity from that from equation (B.1) plotted in Fig 3. The deviation from a harmonic torque was still large as pendulum amplitude increased above about $45^{\circ}$.

\section{Roller pendulum following a circular track}

For a roller pendulum, the quasi-static analysis was essentially the same except that the contact force between the (circular) roller and its (circular) track had to be calculated. If the circumferential component of force at the roller's centre was $H_{1}$, equilibrium of the roller required the tangential component of its contact force to be $\mathrm{H}_{2}$ where:

$$
H_{2}=H_{1}\left(\frac{I}{m r^{2}}\right) /\left(1+\frac{I}{m r^{2}}\right) ;
$$

and the torque equation corresponding to (??) was:

$$
\begin{aligned}
& \frac{T}{m a l \Omega^{2}}=\left\{\frac{1}{1+\frac{I}{m r^{2}}}\right\} \\
& \left\{1+\left(\frac{a}{l}\right) \cos \phi-\left(\frac{r}{l}\right)\left(\frac{I}{m r^{2}}\right)\right\} \sin \phi .
\end{aligned}
$$

Again, a graph of $T / m a L \Omega^{2}$ from (B.8) compares closely in shape with that in Fig 3. A similar deviation from harmonic occurred as pendulum amplitude increased above about $30^{\circ}$.

\section{ADDENDUM}

Bibliography I am grateful to the IJAV's referees of this paper for their helpful comments. One referee particularly mentioned the early contribution of the Italian mathematician Ernesto Cesàro (1859-1906) in describing path geometry in terms of intrinsic curvilinear coordinates which, many years later, would be used effectively in the analysis of centrifugal pendulum devices.

Cesàro, E. Lezioni di geometria intrinseca, published by Italy's National Academy of Sciences, Naples, 1896.

Cesàro, E. Vorlesungen über natürliche Geometrie, German translation by G. Kowalewski, Teubner, Leipzig and Berlin, 1901, 2e 1926.

Although I had not originally intended this paper to be a survey, the referee also gave me a selected list of publications over the last 30 years, and I am pleased to include these below.

Sharif-Bakhtiar, M. and Shaw, S. W. The dynamic response of a centrifugal pendulum vibration absorber with motion-limiting stops, J. Sound Vib., 126(2), 221-235, (1988). https://dx.doi.org/10.1016/0022-460X(88)90237-4

Asfar, K. R. Effect of non-linearities in elastomeric material dampers on torsional vibration control, Int. J. Non-Linear Mechanics, 27(6), 947-954, (1992). https://dx.doi.org/10.1016/0020-7462(92)90047-B

Lee, C. T. and Shaw, S. W. On the counteraction of periodic torques for rotating systems using centrifugally driven vibration absorbers, J. Sound Vib., 191(5), 695-719, (1996). https://dx.doi.org/10.1006/jsvi.1996.0151

Chao, C. P., Lee, C. T., and Shaw, S. W. Nonunison dynamics of multiple centrifugal pendulum vibration absorbers, J. Sound Vib., 204(5), 769-794, (1997).https://dx.doi.org/10.1006/jsvi.1997.0960

Lee, C. T. and Shaw, S. W. The non-linear dynamic response of paired centrifugal pendulum vibration absorbers, J. Sound Vib., 203(5), 731-743, (1997). https://dx.doi.org/10.1006/jsvi.1996.0707
Chao, C. P. and Shaw, S. W. The dynamics response of multiple pairs of subharmonic pendulum vibration absorbers, J. Sound Vib., 231(2), 411-431, (2000https://dx.doi.org/10.1006/jsvi.1999.2722

Steffen, V., and Rade, D. Vibration absorbers, encyclopaedia of vibration, 9-26, Academic Press, San Diego, (2001). https://dx.doi.org/10.1006/rwvb.2001.0176

Alsuwaiyan, A. S. and Shaw, S. W. Performance and dynamic stability of general-path centrifugal pendulum vibration absorbers, J. Sound Vib., 252(5), 791-815, (2002). https://dx.doi.org/10.1006/jsvi.2000.3534

El-Bassiouny, A. F. Effect of non-linearities in elastomeric material dampers on torsional oscillation control, App. Maths and Computation, 162(2), 835-854, (2005). https://dx.doi.org/10.1016/j.amc.2003.12.142

Demeulenaere, B., Spaepen, P., and De Schutter, J. Input torque balancing using a cam-based centrifugal pendulum: design procedure and example, J. Sound Vib., 283(1-2), 1-20, (2005). https://dx.doi.org/10.1016/j.jsv.2004.03.029

$\mathrm{Wu}, \mathrm{S}$. T. Active pendulum vibration absorbers with a spinning support, J. Sound Vib., 323(1-2), 1-16, (2009). https://dx.doi.org/10.1016/j.jsv.2008.12.017

Wenzhi, G. and Zhiyong, Active control and simulation test study on torsional vibration of large turbogenerator rotor shaft, Mechanism and Machine Theory, 45(9), 1326-1336, (2010). https://dx.doi.org/0.1016/j.mechmachtheory.2010.04.005

Wu, S. T., Chen, Y. R., and Wang, S. S. Twodegree-of-freedom rotational-pendulum vibration absorbers, J. Sound Vib., 330(6), 1052-1064, (2011). https://dx.doi.org/10.1016/j.jsv.2010.09.028

Ishida, Y. Recent development of the passive vibration control method, Mech. Systems and Signal Proc., 29,2-18, (2012). https://dx.doi.org/10.1016/j.ymssp.2011.12.005

Wu, S. T. and Siao, P. S. Auto-tuning of a two-degree-of-freedom rotational pendulum absorber, J. Sound Vib.,331(13), 3020-3034, (2012). https://dx.doi.org/10.1016/j.jsv.2012.02.021

Shi, C. and Parker, R. G. Modal properties and stability of centrifugal pendulum vibration absorber systems with equally spaced, identical absorbers, J. Sound Vib., 331(21), 48074824, (2012). https://dx.doi.org/10.1016/j.jsv.2012.05.018

Pfleghaar, J., and Lohmann, B. The electrical dual mass flywheel - an efficient active damping system, IFAC Proceedings, 46(21), 483-488, (2013). https://dx.doi.org/10.3182/20130904-4-JP-2042.00046

Shi, C. and Parker, R. G. Modal structure of centrifugal pendulum vibration absorber systems with multiple cyclically symmetric groups of absorbers, J. Sound Vib., 332(18), 43394353, (2013). https://dx.doi.org/10.1016/j.jsv.2013.03.009

Shi, C., Parker, R. G., and Shaw, S. W. Tuning of centrifugal pendulum vibration absorbers for translational and rotational vibration reduction, Mechanism and Machine Theory, 66, 56-65, (2013). https://dx.doi.org/10.1016/j.mechmachtheory.2013.03.004

Vyhlidal, T., Olgac, N., and Kučera, V. Delayed resonator with acceleration feedback-complete stability analysis by spectral methods and vibration absorber design, J. Sound Vib., 333(25), 6781-6795, (2014).https://dx.doi.org/10.1016/j.jsv.2014.08.002 
Viet, L. D., and Nghi, N. B. On a nonlinear single-mass two-frequency pendulum tuned mass damper to reduce horizontal vibration, Engng Structures, 81, 175-180, (2014). https://dx.doi.org/10.1016/j.engstruct.2014.09.038

Mayet, J., and Ulbrich, H. Tautochronic centrifugal pendulum vibration absorbers: General design and analysis, J. Sound Vib., 333(3), 711-729, (2014). https://dx.doi.org/10.1016/j.jsv.2013.09.042

Issa, J. S., and Shaw, S. W. Synchronous and nonsynchronous responses of systems with multiple identical nonlinear vibration absorbers, J. Sound Vib., 348, 101-125, (2015). https://dx.doi.org/10.1016/j.jsv.2015.03.021

Habib, G., Detroux, T., Viguié, R., and Kerschen, G. Nonlinear generalization of Den Hartog's equal-peak method, Mech. $\quad$ Systems and Signal Proc., 52, 1-28, (2015). https://dx.doi.org/10.1016/j.ymssp.2014.08.009

Shi, C., Shaw, S. W., and Parker, R. G. Vibration reduction in a tilting rotor using centrifugal pendulum vibration absorbers, J. Sound Vib., 385, 55-68, (2016). https://dx.doi.org/10.1016/j.jsv.2016.08.035

Mall, P., Fidlin, A., Krüger, A., andGross, H. Simulation based optimization of torsional vibration dampers in automotive powertrains, Mechanism and Machine Theory, 115, 244-266, (2017). https://dx.doi.org/10.1016/j.mechmachtheory.2017.05.010

Haris, A., Motato, E., Mohammadpour, M., Theodossiades, S., Rahnejat, H., O' Mahony, M., and McFarland, D. M., On the effect of multiple parallel nonlinear absorbers in palliation of torsional response of automotive drivetrain, Int. J. Non-Linear Mechanics, 96, 22-35, (2017). https://dx.doi.org/10.1016/j.ijnonlinmec.2017.06.008

Karmazyn, A., Balcerzak, M., Perlikowski, P., and Stefanski, A. Chaotic synchronization in a pair of pendulums attached to driven structure, Int. J. Non-Linear Mechanics, 105, 261-267, (2018). https://dx.doi.org/10.1016/j.ijnonlinmec.2018.05.013

Sun, C., and Jahangiri, V. Bi-directional vibration control of offshore wind turbines using a 3D pendulum tuned mass damper, Mech. Systems and Signal Proc., 105, 338-360, (2018). https://dx.doi.org/10.1016/j.ymssp.2017.12.011

Gao, P., Xiang, C., Liu, H., Walker, P., and Zhang, N. Design of the frequency tuning scheme for a semi-active vibration absorber, Mechanism and Machine Theory, 140, 641-653, (2019). https://dx.doi.org/10.1016/j.mechmachtheory.2019.06.025

Bab, S., Najafi, M., Fathi Sola, J., and Abbasi, A. Annihilation of non-stationary vibration of a gas turbine rotor system under rub-impact effect using a nonlinear absorber, Mechanism and Machine Theory, 139, 379-406, (2019). https://dx.doi.org/10.1016/j.mechmachtheory.2019.05.005

Cirelli, M., Gregori, J., Valentini, P. P., and Pennestrí, E. A design chart approach for the tuning of parallel and trapezoidal bifilar centrifugal pendulum, Mechanism and Machine Theory, 140, 711-729, (2019). https://dx.doi.org/10.1016/j.mechmachtheory.2019.06.030

Stefanski, A., Pikunov, D., Balcerzak, M., and Dabrowski, A. Synchronized chaotic swinging of parametrically driven pendulums, Int. J. Mech. Sci., 173, 105454, (2020). https://dx.doi.org/10.1016/j.ijmecsci.2020.105454
It will be a pleasure to see this paper published in the IJAV, a quarter of a century after I helped start the journal and over half a century after my first publication on this subject in 1964.

\section{REFERENCES}

1 Alsuwaiyan, A. S., and Shaw, S. W. Performance and dynamic stability of general-path centrifugal pendulum vibration absorbers, J. Sound Vib., 252(5), 791-815, (2002). https://dx.doi.org/10.1006/jsvi.2000.3534

2 Chao, C. -P., Lee, C. -T. and Shaw, S. W. Nonunison dynamics of multiple centrifugal pendulum vibration absorbers, J. Sound Vib, 204(5), 769-794, (1997).https://dx.doi.org/10.1006/jsvi.1997.0960

3 Den Hartog, J. P. Mechanical vibrations, 4th ed., McGrawHill, New York, 1956.

4 Denman, H. H. Tautochronic bifilar pendulum torsion absorbers for reciprocating engines, J. Sound Vib, 159(2), 251-277, (1992). https://dx.doi.org/10.1016/0022460X(92)90035-V

5 Ishida, Y., Inoue, T., Fukami, T. and Ueda, M. Torsional vibration suppression by roller type centrifugal vibration absorbers, J. Vib. Acoustics, 131(5), 10 pages, (2009).https://dx.doi.org/10.1115/1.3147124

${ }^{6}$ Lee, C.-T. and Shaw, S. W. On the counteraction of periodic torques for rotating systems using centrifugally driven vibration absorbers, J. Sound Vib., 191(5), 695-719, (1996). https://dx.doi.org/10.1006/jsvi.1996.0151

7 McKinley, J. M. Brachistochrones, tautochrones, evolutes and tesselations, Am. J. Physics, 47(1), 81-86, (1979). https://dx.doi.org/10.1119/1.11679

8 Mitchiner, R. G. and Leonard, R. G. Centrifugal pendulum vibration absorbers-theory and practice, J. Vib. Acoustics, Trans. ASME, 113(4), 503-507, (1991). https://dx.doi.org/10.1115/1.2930214

9 Newland, D. E. Nonlinear vibrations: A comparative study with applications to Centrifugal Pendulum Vibration Absorbers, ScD thesis, Massachusetts Institute of Technology, 1963.

10 Newland, D. E. Nonlinear aspects of the performance of centrifugal pendulum vibration absorbers, J. of Eng. for Industry, Trans. ASME, Series B, 86 (3), 257-263, (1964). https://dx.doi.org/10.1115/1.3670529

11 Newland, D. E. Nonlinear problems of centrifugal pendulum vibration absorbers, Proc. Int. Conf. on Mechanisms and Machines, Varna, Bulgaria, (1965). http://www2.eng.cam.ac.uk/ den/papers/DEN\%20Varna $\% 201965 \% 20$ Paper.pdf

12 Shaw, S. W., and Geist, B. Tuning for performance and stability in systems of nearly tautochronic torsional vibration absorbers, J. Vib. \& Acoustics, ASME, 132 (4), 11 pages, (2010). https://dx.doi.org/10.1115/1.4000840

13 Shaw, S. W., Schmitz, P. M., and Haddow, A. G. Tautochronic vibration absorbers for rotating systems, J. Comp. and Nonlinear Dynamics, 1(4), 283-293, (2006). https://dx.doi.org/10.1115/1.2338652 